\title{
Anisotropic distribution of space pulsar velocities
}

\author{
Igor Fedorovich Malov \\ P. N. Lebedev Physical Institute, Russian Academy of Sciences, Pushchino, Russia
}

\section{Email address:}

malov@prao.ru

\section{To cite this article:}

Igor Fedorovich Malov. Anisotropic Distribution of Space Pulsar Velocities. American Journal of Astronomy and Astrophysics. Special Issue: Global Anisotropy, Theory of Byuon, New Force, New Power System, Propulsion, Space Flights. Vol. 2, No. 6-1, 2014, pp. 29-31.

doi: 10.11648/j.ajaa.s.2014020601.14

\begin{abstract}
On the base of new data it is shown that the observed proper motions and directions of the tangential velocities of pulsars manifest the extremely anisotropic distributions. These anisotropies cannot be explained by the structure of our Galaxy or by various types of solar motions. They can be caused by the cosmological vector potential and the action of the new force. This force must be stronger in higher magnetic fields. The positive correlation between tangential velocities $V_{t}$ and surface magnetic fields of pulsars $B_{s}$ is seen indeed. It is shown that the new force can provide pulsar velocities up to several thousands of $\mathrm{km} / \mathrm{sec}$.
\end{abstract}

Keywords: Pulsars, Proper Motions, Magnetic Fields, New Force

\section{Introduction}

A radio pulsar can be considered as one of the most exciting astrophysical objects. Indeed its central body is a neutron star with radii of order of $10 \mathrm{~km}$ and masses of order of the solar mass $\left(2 \times 10^{33} \mathrm{~g}\right)$, the nuclear density $\left(10^{14}-10^{15}\right.$ $\mathrm{g} / \mathrm{cm}^{3}$ ), superfluid neutrons and superconducting protons in inner regions and magnetic fields of order of $10^{12} \mathrm{G}$ at the surface.There are relativistic electrons and positrons in magnetospheres of neutron stars.These stars are formed during explosions of supernovae. Many unusual physical processes are realized in radio pulsars, and they can be used to verify some new theories and models. To better understand the origin and evolution of radio pulsars, it is very important to know the space velocities of these objects and the distribution of these velocities. Unfortunately, the absence in pulsars of spectral lines that could be compared to the laboratory standards makes estimation of their radial velocities impossible.Therefore, studies are basically limited to tangential velocities derived from observed proper motions of the pulsars in the plane of the sky. Corresponding values can be found in the pulsar catalogue [1]. This moment it contains parameters of more than 2300 objects, however proper motions have been measured for approximately 200 pulsars only.

The velocities of pulsars were found to be very high, in some cases, exceeding $1000 \mathrm{~km} / \mathrm{s}$ (see, e.g., [2] and references therein). Several mechanisms have been proposed to explain such high velocities, and are reviewed, for example, by Lai [3].

\section{Proper Motions of Radio Pulsars}

First of all we shall analyse measured proper motions from the catalogue [1]. Using the angle between the motions in right ascension $\mu_{\alpha}=(\mathrm{d} \alpha / \mathrm{dt}) \operatorname{Cos} \delta$ and declination $\mu_{\delta}=\mathrm{d} \delta / \mathrm{dt}$ :

$$
\xi=\operatorname{atn}\left(\mu_{\alpha} / \mu_{\delta}\right)
$$

we obtain the distribution of the directions of such motions in the plane of the sky.

Here $\alpha$ is the right ascension and $\delta$ the declination of the pulsar considerd. Maximal values of $\mu_{\alpha}$ and $\mu_{\delta}$ are of order of 100 marcsec, therefore we can use the plane trigonometry to calculate $\xi$ (Fig.1)[4]

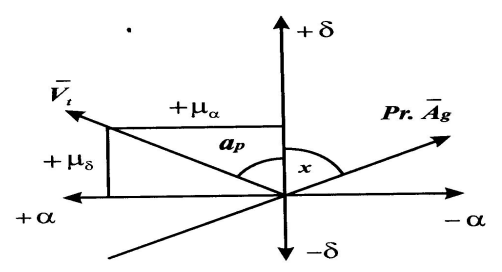

Fig 1. Direction of a pulsar motion $\left(V_{t}\right)$ on the plane of the sky, vector $A_{g}$ (cosmological vector potential) will be described further. 
Apriori we must expect that the space velocities of pulsars when they are formed are randomly directed. Therefore, the distribution of the directions of pulsar motions should be isotropic. However Fig.2-3 show that directions of pulsar motions are distributed very ununiformly.

The structure of the Galaxy and the pattern of its magnetic fields cannot cause this anisotropy. It can likewise not be explained by the acceleration of binary systems due to asymmetric X-ray radiation because of deviations of the neutron-star magnetic field from a dipolar field [5].

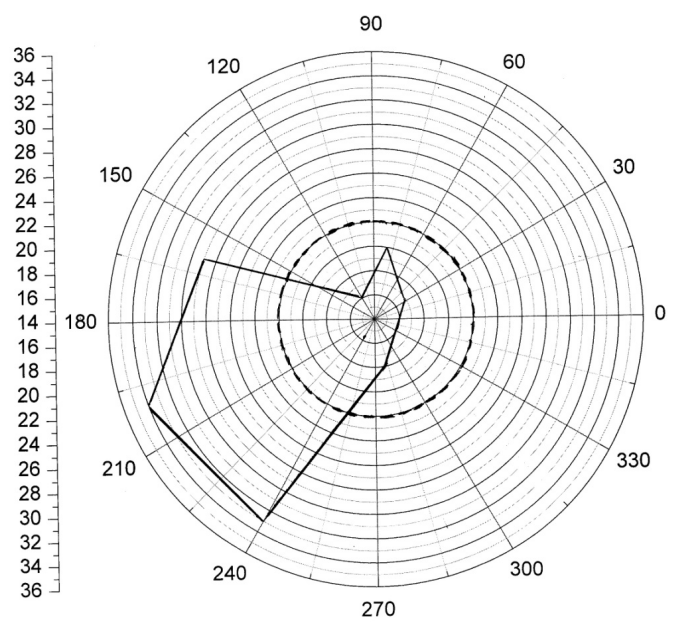

Fig 2. Distribution of observed angles $\xi$ (solid line). Dashed line shows the expected uniform distribution.

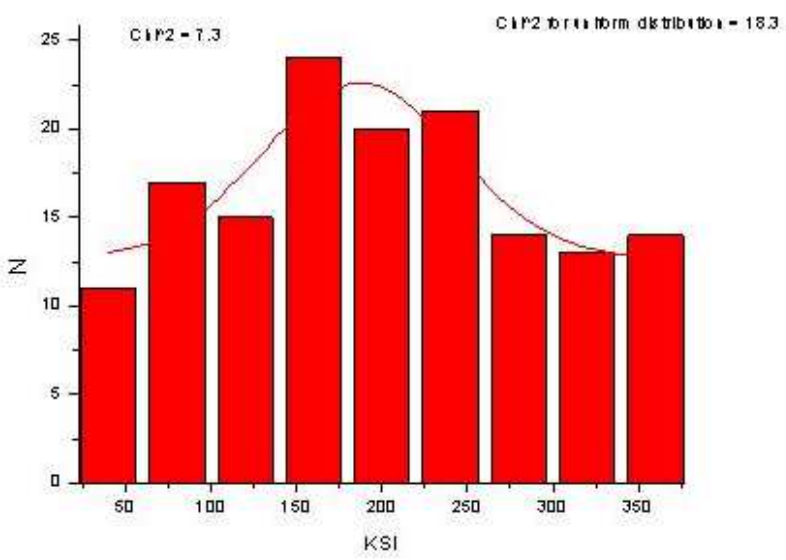

Fig 3. Histogram representing the distribution of angles $\xi$.

\section{Distribution of Pulsar Velocities}

It is quite interesting to determine the main direction of pulsar motions. We estimated angles between transverse velocities of pulsars $\mathrm{V}_{\mathrm{t}}$ and «the cosmological» vector potential $A_{g}$ [6]. For this aim we must use spherical trigonometry (Fig.4) [4].

We need to calculate the angle $\varepsilon$. The system of equations for our problem has the following form [7]:

$$
\begin{gathered}
\operatorname{Cos} \mathrm{z}=\operatorname{Sin} \delta_{\mathrm{PSR}} \operatorname{Sin} \delta_{\mathrm{Ag}}+\operatorname{Cos} \delta_{\mathrm{PSR}} \operatorname{Cos} \delta_{\mathrm{Ag}} \operatorname{Cos}\left(\alpha_{\mathrm{Ag}}-\alpha_{\mathrm{PSR}}\right) \\
\operatorname{Sin} \mathrm{x}=\operatorname{Cos} \delta_{\mathrm{Ag}} \operatorname{Sin}\left(\alpha_{\mathrm{Ag}}-\alpha_{\mathrm{PSR}}\right) / \operatorname{Sin} \mathrm{z}
\end{gathered}
$$

$$
\operatorname{Cos} \varepsilon=\operatorname{Sin} z \operatorname{Cos}(x+\xi)
$$

The distribution of angles $\varepsilon$ between $V_{t}$ and $A_{g}$ is shown in Fig.5.and 6 As we can see there is the preferable direction of pulsar motion near $120^{\circ}$ from the direction of $A_{g}$. In any case the uniform distribution is very bad approximation of the observed picture.

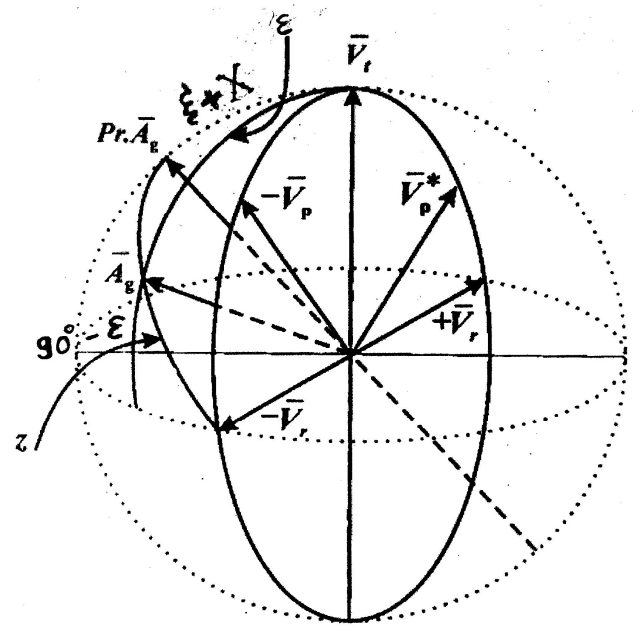

Fig 4. Distribution of different angles on the celestial sphere.

\section{Pulsar Velocities and the New Force}

We use the formula (2) from [4]:

$$
\mathrm{V}=\left(2 \mathrm{~m}_{v} \mathrm{c}^{2} / \mathrm{m}_{\mathrm{n}}\right) \lambda_{1}^{2} \Delta \mathrm{A}(\Delta \mathrm{A} / \Delta \mathrm{X}) \mathrm{T}
$$

where $\mathrm{T}$ is time of action of the new force, $2 \mathrm{~m}_{\mathrm{v}} \mathrm{c}^{2}=33$ $\mathrm{eV}$, the coefficient $\lambda_{1}=10^{-12}(\mathrm{G} \mathrm{cm})^{-1}$ [2], $\Delta \mathrm{A}=10^{11} \mathrm{G} \mathrm{cm} \mathrm{[2],}$ $\Delta \mathrm{A} / \Delta \mathrm{X}$ is the magnetic field at the surface of the pulsar, . $\mathrm{m}_{\mathrm{n}}$ is the mass of neutron. This expression means that $\mathrm{V}$ is proportional to magnetic field $\mathrm{B}$. Fig 7 shows that such a tendensy is observed indeed.

In Fig.7 pulsars in binary systems were excluded, because some uninvestigated processes could play a role in a distortion of the dependence (5).

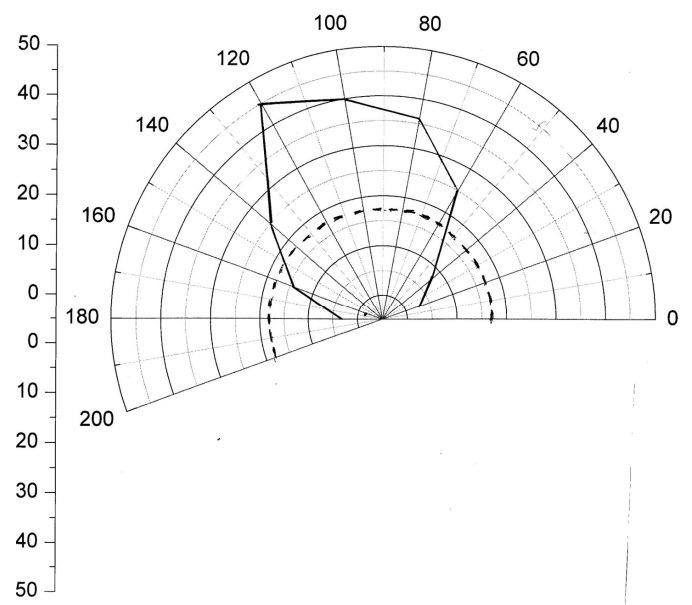

Fig 5. Distribution of angles between $V_{t}$ and $A_{g}$ (solid line). Dashed line is the iniform distribution. 


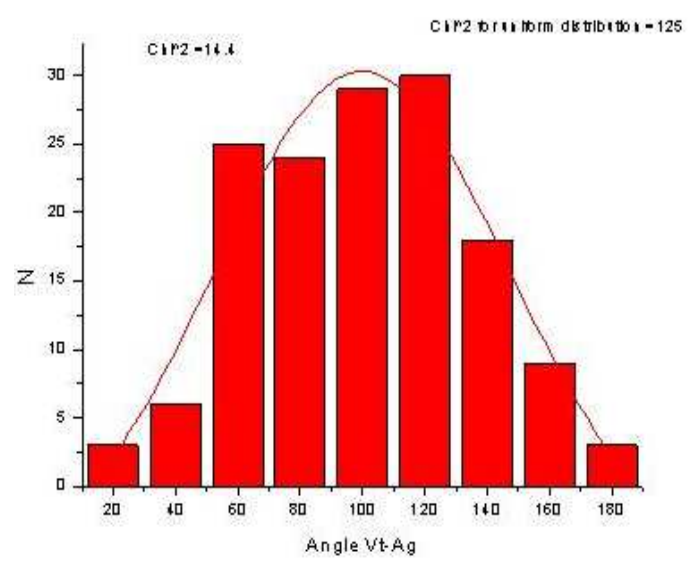

Fig 6. Alternative presentation of the tistribution of angles $\varepsilon$.

Magnetic fields at the pulsar surfaces can achieve values more than $10^{12} \mathrm{G}$. For such fields and the used parameters we can obtain from (5) velocities up to several thousands $\mathrm{km} / \mathrm{sec}$.

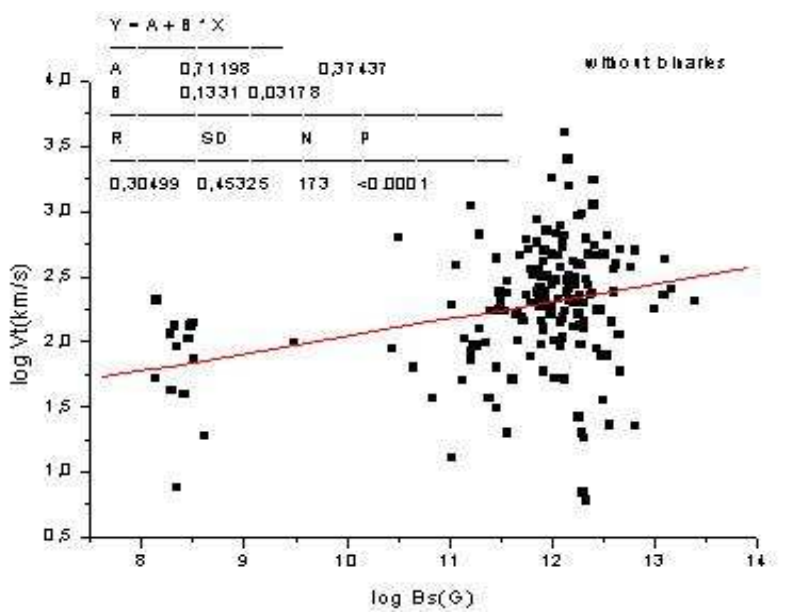

Fig 7. Dependence of the transverse velocities of pulsars on their surface magmetic fields.

\section{Conclusions}

1. New data confirm the previous conclusion on the extremely anisotropic distributions of the observed proper motions and directions of the tangential velocities of pulsars. Let us emphasize that former results have been obtained on the base of data for several dozens of pulsars (for example, 45 objects in [2]). The confirmation of these results shows that the anisotropy of pulsar velocities is real.

2. The observed anisotropies can be caused by the cosmological vector potential.

3. The new force can provide pulsar velocities up to several thounds $\mathrm{km} / \mathrm{sec}$.

4. The action of the new force must be stronger in higher magnetic fields. There is indeed the positive correlation between $V_{t}$ and $B_{s}$.

\section{References}

[1] R.N.Manchester, G.B.Hobbs, A.Teoh, and M.Hobbs. The Australia Telescope National Facility Pulsar Catalogue. Astron.J., vol.129, pp.1993-2006, 2005.

[2] Z.Arzoumanian, D.F.Chernoff, and J.M.Cordes. The Velocity Distribution of Isolated Radio Pulsars Astrophys.J.,vol.289301, pp.568, 2002.

[3] D. Lai. Neutron star kicks and supernova asymmetry..In Cosmic Explosions in Three Dimensions: Asymmetries in Supernovae and Gamma-Ray Bursts, Ed. by P. Hoflich, P. Kumar, and J. C. Wheeler (Cambridge Univ. Press, Cambridge, 2004), p. 276; astro-ph/0312542.

[4] Yu.A.Baurov, A.A.Shpitalnaya, and I.F.Malov. Global Anisotropy of Physical Space and Velocities of Pulsars. Int. J.of Pure and Applied Physics. vol..1, №1, pp..71-82, 2005.

[5] V. D. Pal'shin and A. I. Tsygan. Acceleration of binary X-ray sources by their radiation. Astron. Lett., vol. 24, pp.131133,1998

[6] Yu.A.Baurov. On the structure of physical vacuum and a new interaction in Nature (Theory, Experiment and Applications), Nova Science, NY, 2000.

[7] I.F.Malov, Yu.A.Baurov. On the Distribution of Radio Pulsars Velocities.Int. J. of Pure and Applied Physics. vol.2, №3, pp.273-280, 2006. 